\title{
Clearance of Bone Substitute in Gel Form Accidentally Dispersed into the Sinus Cavity during Transcrestal Maxillary Sinus Floor Elevation: Two-Case Report
}

\author{
Fabio Bernardello ${ }^{1}$, Teresa Lombardi ${ }^{2}$ and Claudio Stacchi ${ }^{3, *}$ (D) \\ 1 Private Practice, 37045 Terranegra di Legnago, Italy; fabio.tredici@libero.it \\ 2 Department of Health Sciences, Magna Græcia University, 88100 Catanzaro, Italy; drteresalombardi@libero.it \\ 3 Department of Medical, Surgical and Health Sciences, University of Trieste, 34100 Trieste, Italy \\ * Correspondence: claudio@stacchi.it; Tel.:+39-048-153-1229
}

Citation: Bernardello, F.; Lombardi, T.; Stacchi, C. Clearance of Bone Substitute in Gel Form Accidentally Dispersed into the Sinus Cavity during Transcrestal Maxillary Sinus Floor Elevation: Two-Case Report. Sinusitis 2021, 5, 132-140. https:// doi.org/10.3390/sinusitis5020014

Academic Editor: Sara Bernardi

Received: 6 September 2021

Accepted: 25 October 2021

Published: 27 October 2021

Publisher's Note: MDPI stays neutral with regard to jurisdictional claims in published maps and institutional affiliations.

\section{Copyright: (๑) 2021 by the authors.} Licensee MDPI, Basel, Switzerland. This article is an open access article distributed under the terms and conditions of the Creative Commons Attribution (CC BY) license (https:// creativecommons.org/licenses/by/ $4.0 /)$.

\begin{abstract}
Sinus membrane perforation is the most frequent intraoperative complication occurring during maxillary sinus floor elevation. Although numerous techniques for perforation management are present, grafting material dissemination may still occur, representing a potential trigger factor leading to acute or chronic sinusitis. This case report describes two cases of xenogeneic bone substitute in gel form accidentally dispersed into the sinus cavity during maxillary sinus floor elevation with a transcrestal approach. In both cases, immediately postoperative radiographic imaging showed an important amount of gel graft dislodged into the sinus cavity as a consequence of hidden perforations that remained undetected during surgery. Patients were monitored for 6 months after surgery and reported no signs or symptoms related to possible sinus disease. Control radiographs showed no sinus membrane hypertrophy and/or presence of residual disseminated gel, confirming complete clearance of the accidentally dispersed graft through the ostiomeatal complex. In order to minimize postoperative complications, bone substitutes in gel form could represent an interesting alternative to granular grafts for their easier clearance from the maxillary sinus cavity in case of accidental dissemination during sinus augmentation procedures.
\end{abstract}

Keywords: maxillary sinus; maxillary sinus floor elevation; foreign-body sinusitis; bone substitutes; grafting materials

\section{Introduction}

Schneiderian membrane perforation represents the most frequently described intraoperative complication occurring during maxillary sinus floor elevation. The incidence of this adverse event has been reported to vary between $11 \%$ and $20 \%$ for the lateral tech-nique and from $0 \%$ to $20 \%$ for the transcrestal approach [1-3]. Proper management of perforation is mandatory in order to complete membrane elevation and graft placement. Many approaches have been proposed in literature to overcome this problem, including suturing techniques, or the use of resorbable membranes, demineralized lamellar bone, biological glues, and buccal fat pad flap [4-8]. Nevertheless, particulate grafting material displacement into the sinus cavity remains a possible adverse event in case of Schneiderian membrane perforation, even after attempting to seal it during surgery [9].

Both autologous bone and various bone substitutes have been studied in the last decades as grafting materials for maxillary sinus floor elevation. A recent meta-analysis including more than 130 studies with histomorphometric data showed that autologous bone resulted in the highest amount of newly formed bone but its use is associated to significant drawbacks (high resorption rate, limited availability, and patient morbidity) [10]. Bone substitutes (allografts, xenografts, and alloplastic materials) appear to be good alternatives to autologous bone both in terms of osteoconductive properties and long-term survival rate 
of dental implants inserted in augmented sinuses [11,12]. For this application, bone substitutes are available in different formulations: granules, blocks, paste, and gel. Allografts and xenografts in granules, the most commonly used in the clinical practice, are available with different characteristics both in composition (cortical, cortico-cancellous, or cancellous) and in graft particle dimensions. These materials undergo a very slow remodeling process: residual particles are recognizable in the regenerated sites up to 20 years after grafting procedure [13,14]. In case of sinus membrane perforation, bone substitutes in granular form could be disseminated into the maxillary sinus cavity, possibly compromising ostiomeatal complex $(\mathrm{OMC})$ patency and favoring the onset of foreign body sinusitis $[15,16]$. In the case of postoperative infection associated with graft dispersion into the sinus cavity, recent guidelines suggest a multidisciplinary approach to manage the complication [17]. Functional endoscopic sinus surgery (FESS) could be necessary, in combination with the removal of grafting material and dental implants with an oral approach [18].

Recently, xenogeneic and alloplastic bone substitutes in gel form have been proposed in literature as grafting material for transcrestal sinus floor elevation [19-22]. These biomaterials are composed by micronized particles (generally up to $300 \mu \mathrm{m}$ ) suspended in an aqueous solution and embedded in a collagen matrix. These biomaterials are easily injectable into the subantral space after performing a crestal access to the sinus cavity through the cortical of the sinus floor. Micronized particles are smooth and do not present sharp or cutting edges, potentially representing a risk factor for sinus membrane tearing during grafting procedures. Additionally, bone substitutes in gel form accidentally dispersed into the sinus cavity could be more easily cleared through the OMC by the ciliary activity, due to the pasty consistency and the extremely small dimensions of the particles.

The present report describes two cases of sinus membrane perforation during transcrestal sinus floor elevation by using grafts in gel form and the clinical outcomes following accidental membrane tearing and graft dispersion into the sinus cavity.

\section{Case Description}

\subsection{Patient \#1}

A 77 year old female patient presented to our observation, requiring an implantsupported rehabilitation for partial edentulism of the posterior maxilla (first and second upper right molar). Presurgical radiographic investigations (cone beam computerized tomography, $\mathrm{CBCT}$ ) revealed limited vertical bone dimension in the programmed implant sites, with residual bone height (RBH) of $6.8 \mathrm{~mm}$ in the mesial site and $6.4 \mathrm{~mm}$ in the distal position. No signs of sinus membrane hypertrophy were present (Figure 1a,b).

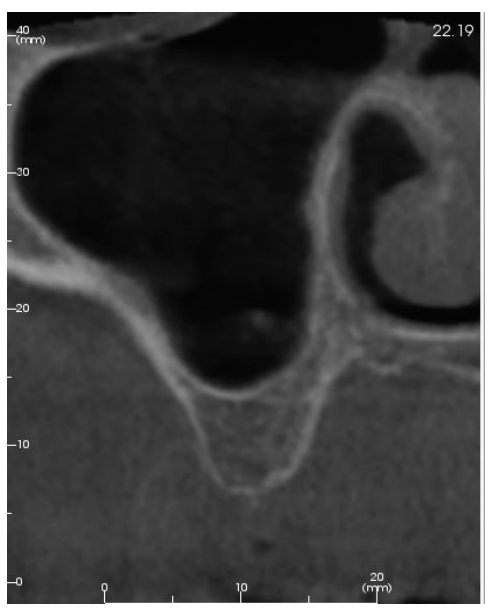

(a)

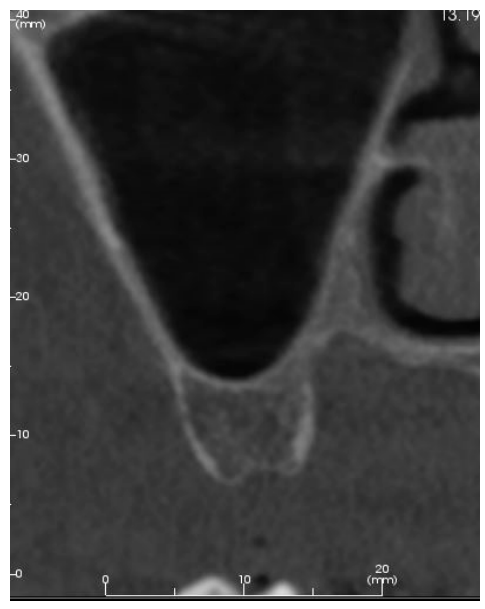

(b)

Figure 1. Patient \#1. Preoperative CBCT cross-section images of the surgical sites showed: (a) first molar area: adequate ridge width and $6.8 \mathrm{~mm}$ available bone height; (b) second molar area: adequate 
ridge width and $6.4 \mathrm{~mm}$ available bone height. Schneiderian membrane showed no signs of hypertrophy or inflammation.

$\mathrm{RBH}$ was not sufficient for the insertion of standard length implants, therefore a sinus augmentation procedure was programmed. Narrow sinus anatomy suggested the possibility to perform a transcrestal technique [23], less invasive if compared with a lateral approach; additionally, $\mathrm{RBH}$ (>3 mm) was sufficient to allow implant insertion contextually to the augmentation procedure [24].

One hour before surgery, the patient was administered with amoxicillin/clavulanate acid ( $2 \mathrm{~g}$ single-dose), as antibiotic prophylaxis. After local infiltration of anesthetic solution (articaine hydrochloride, 4\% with 1/100,000 adrenaline, Septanest, Septodont, Saint-Maur Des Fosses, France), a minimally invasive full-thickness flap was elevated. Osteotomy and crestal sinus access were performed using specific sequential drills (Crestal Sinus Lift, i-Res Dental, Mendrisio, Switzerland) [25]. These particular drills are used in a sequence of increasing length, until reaching the sinus floor; the smooth cutting angle of drills allows to complete sinus floor osteotomy without damaging the sinus membrane. After checking membrane integrity with Valsalva maneuver, micronized collagenated xenograft in gel form (Osteobiol Gel 40, Roen, Pianezza, Italy) was slowly injected under the sinus membrane until creating sufficient subantral space to insert dental implants of adequate length (i-MAX Hybrid, i-Res Dental, Mendrisio, Switzerland). Two implants were inserted in the surgical sites, achieving a primary stability of $30 \mathrm{Ncm}$, and flaps were sutured with single stitches to allow submerged implant healing. The patient was prescribed with antibiotics for 6 days (amoxicillin/clavulanate acid $1 \mathrm{~g}$ three times a day) and nonsteroidal anti-inflammatory drugs (ibuprofen $600 \mathrm{mg}$ ), when needed.

\subsection{Patient \#2}

A 73 year old female patient required an implant-supported rehabilitation for partial edentulism, with missing first and second upper right premolars and first upper right molar. Presurgical diagnostic investigations (CBCT) showed an adequate bone volume in the first premolar area but a limited $\mathrm{RBH}$ in the second premolar $(2.7 \mathrm{~mm})$ and first molar $(1.2 \mathrm{~mm})$ surgical sites. The Schneiderian membrane showed no signs of hypertrophy or inflammation (Figure 2a,b).

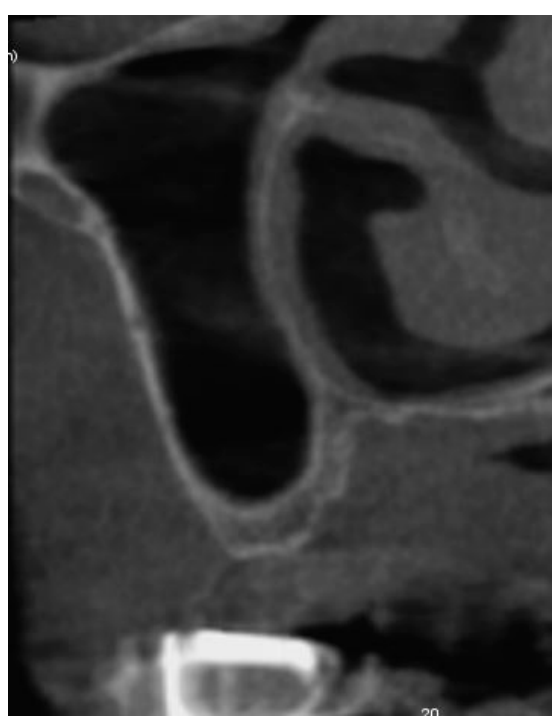

(a)

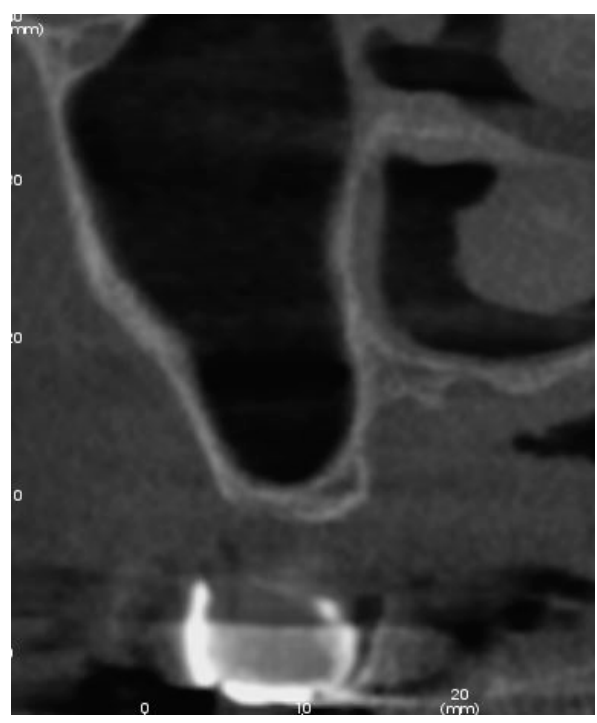

(b)

Figure 2. Patient \#2. Preoperative CBCT cross-section images of the surgical sites showed: (a) second premolar area: adequate ridge width and $2.7 \mathrm{~mm}$ available bone height; (b) first molar area: adequate 
ridge width and $1.2 \mathrm{~mm}$ available bone height. Schneiderian membrane showed no signs of hypertrophy or inflammation.

In these last sites RBH was not sufficient for the insertion of dental implants of adequate length, therefore only maxillary sinus floor elevation was planned. The narrow sinus anatomy at the edentulous sites allowed to perform a transcrestal approach; however, $\mathrm{RBH}<3 \mathrm{~mm}$ suggested delayed implant placement [24].

The surgical procedure was performed exactly as described in Section 2.1, with the only exception that dental implants were not placed after graft injection.

\section{Results}

\subsection{Patient \#1}

\subsubsection{Surgery}

Immediately after surgery, a low-dose $\mathrm{CBCT}$ check was performed. Cross-section images (Figure 3a,b) showed regular "dome-shaped" membrane elevation with graft surrounding both implant apexes. The procedure appeared regularly performed: however, the evaluation of the Panorex image revealed a perforation in the distal apical portion of the dome generated by graft injection. An important amount of graft in gel form dispersed into the sinus cavity was easily detectable, also for its evident radiopacity (Figure 3c). The patient was discharged, with the recommendation to avoid nose blowing and to sneeze with mouth open to limit the risk of increasing graft dispersion through the perforation.

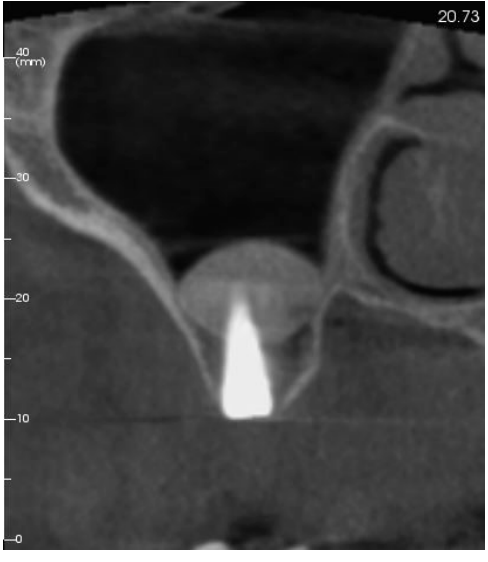

(a)

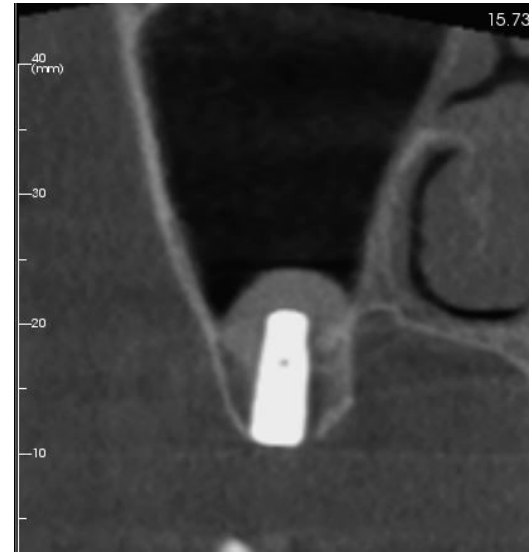

(b)

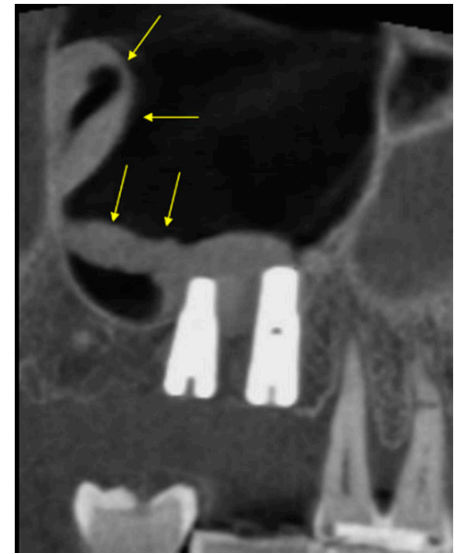

(c)

Figure 3. Patient \#1. Immediately postoperative CBCT showing: $(\mathbf{a}, \mathbf{b})$ cross-section images of the surgical sites with regular "dome-shaped" membrane elevation and graft surrounding both implant apexes; (c) Panorex view highlighting a perforation in the distal apical portion of the elevated area, with arrows indicating biomaterial dispersed into the sinus cavity.

\subsubsection{Follow Up}

The patient was monitored every two weeks and reported no signs and/or symptoms referable to a possible sinusitis during the early healing period. A low-dose CBCT was performed 2 months after surgery to check the evolution of the complication. Both crosssection and Panorex images (Figure $4 a-c$ ) still showed graft surrounding implants apexes but also a diffuse, almost complete radiopacity of the right sinus; no residual gel graft dispersed into the sinus cavity was detectable. 


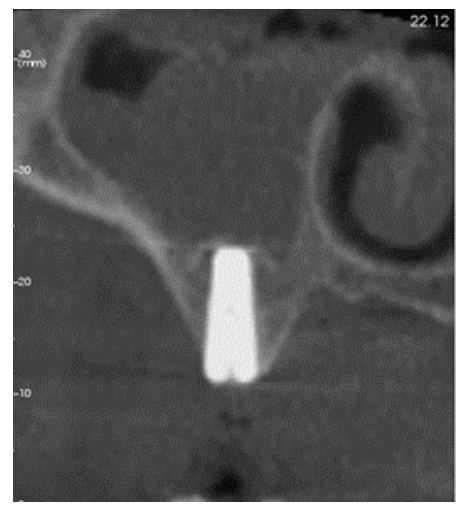

(a)

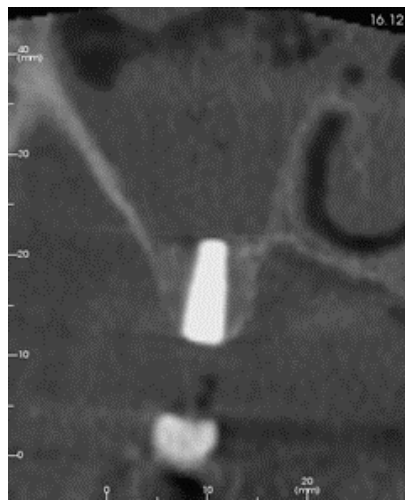

(b)

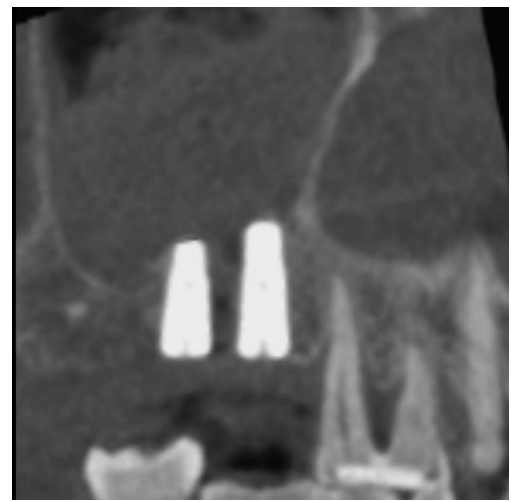

(c)

Figure 4. Patient \#1. CBCT performed 2 months after surgery showing: $(\mathbf{a}, \mathbf{b})$ cross-section images of the surgical sites with graft still surrounding both implant apexes; (c) Panorex view showing almost complete sinus opacification without evidence of residual grafting material disseminated into the cavity.

Implants were connected to healing abutments 6 months after surgery and, after soft tissue maturation, were loaded with two screw-retained metal-porcelain crowns. A lowdose CBCT check, acquired after 6 months of function, showed regular remodeling of the gel graft with newly formed bone in the areas of transcrestal sinus lift: a new cortical layer was clearly detectable at the top of the implants. No radiopacity or residual disseminated gel graft were observable, confirming restored maxillary sinus homeostasis and complete clearance of the accidentally dispersed graft (Figure $5 a-c)$.

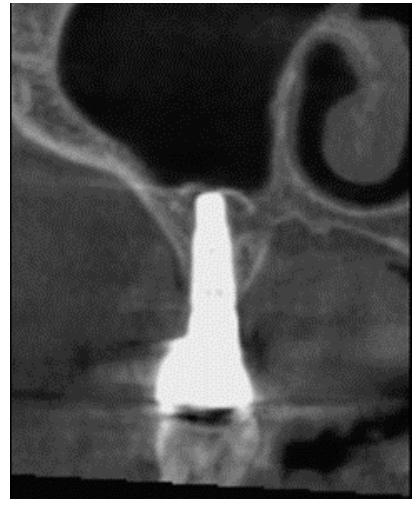

(a)

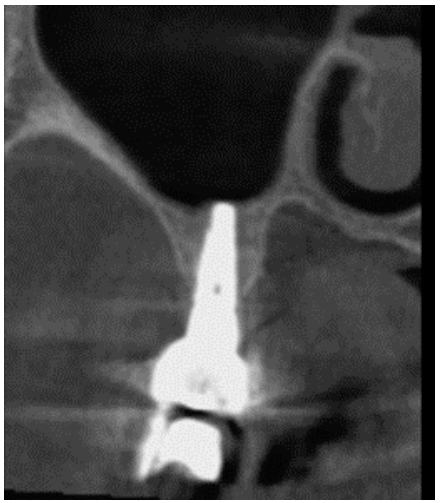

(b)

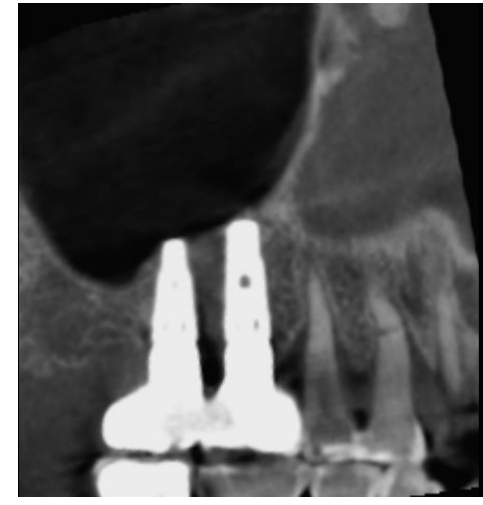

(c)

Figure 5. Patient \#1. CBCT performed after 6 months of functional loading of the implants, showing graft maturation and the formation of a new cortical layer at the top of the implants. No radiopacity or residual graft disseminated into the sinus cavity were observable in cross-section $(\mathbf{a}, \mathbf{b})$ or in Panorex (c) images.

\subsection{Patient Two \\ 3.2.1. Surgery}

Immediately after surgery a low-dose $\mathrm{CBCT}$ was performed to evaluate the procedure. Cross-section and Panorex images (Figure 6a-c) showed that a membrane perforation occurred in the intermediate site (second premolar), whereas a regular "dome-shaped" lift of the membrane was obtained in the more distal and atrophic site (first molar). The dispersed gel graft was appreciable in the sinus cavity without any involvement of the regularly lifted second site. The patient was discharged with the usual behavioral recommendations for the postoperative period. 


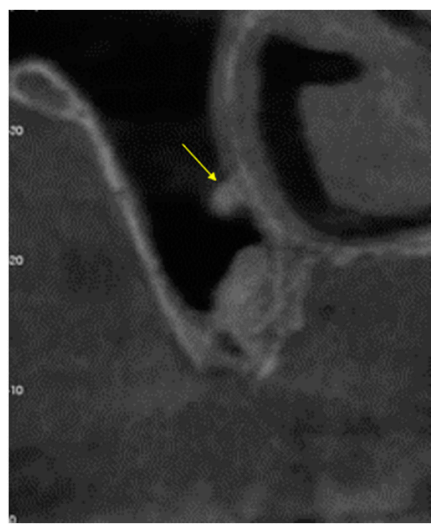

(a)

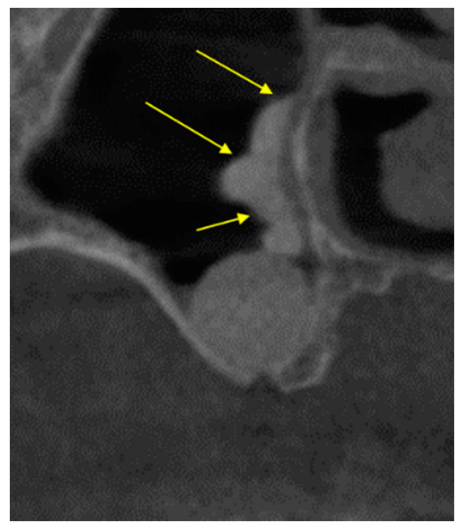

(b)

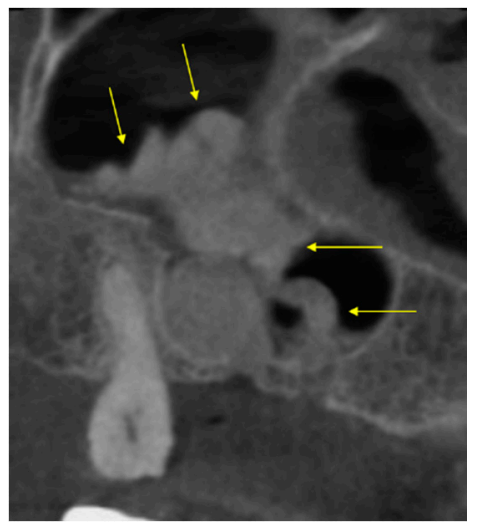

(c)

Figure 6. Patient \#2. Immediately postoperative CBCT showing membrane perforation occurred at the second premolar site (a), whereas a regular "dome-shaped" lift of the membrane was obtained in the first molar area (b). Panorex (c) shows a significant amount of gel graft dispersed into the sinus cavity through a membrane perforation remained undetected during surgery. Dispersed biomaterial is indicated in $(\mathbf{a}-\mathbf{c})$ by the arrows.

\subsubsection{Follow Up}

The patient was monitored every two weeks for the first two months and then every 30 days for the following 4 months. No signs and/or symptoms referable to a possible sinusitis were reported or detected during the entire healing period.

A low-dose CBCT check was performed 6 months after surgery to plan implants insertion. CBCT showed a regular remodeling of the gel graft into new bone in the first molar area: vertical available ridge height was now increased to $8.6 \mathrm{~mm}$, starting from $\mathrm{RBH}$ of $1.2 \mathrm{~mm}$ at baseline (Figure 7a). No new bone formation was detectable in the second premolar site (where the perforation occurred) and no radiographic evidence of disseminated residual biomaterial was observable, confirming the complete clearance of the accidentally dispersed gel graft (Figure $7 \mathrm{~b}$ ).

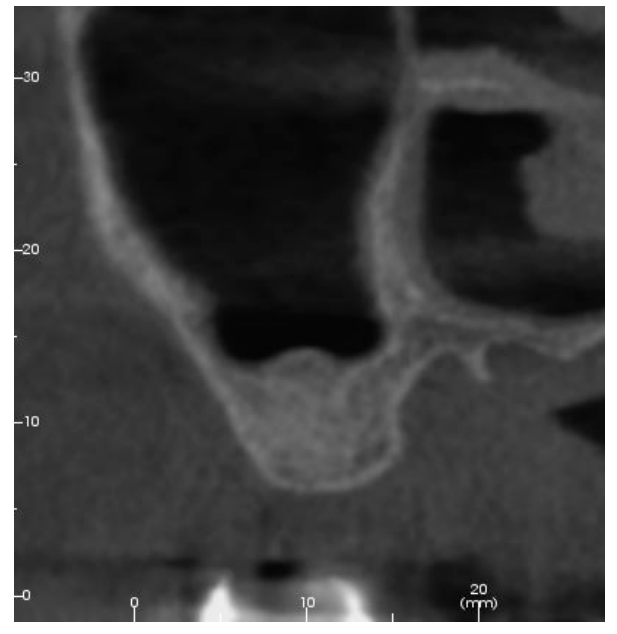

(a)

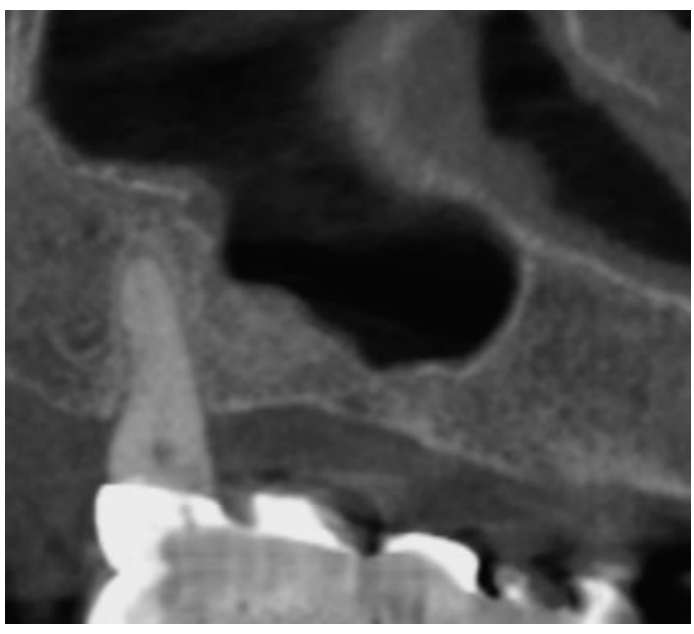

(b)

Figure 7. Patient \#2. Postoperative CBCT cross-section image of the first molar area showing regular remodeling of the gel graft into new bone with vertical available ridge height of $8.6 \mathrm{~mm}$ (a). No radiographic evidence of disseminated residual biomaterial was observable in the Panorex view, with sinus membrane showing no signs of hypertrophy or inflammation (b). 


\section{Discussion}

In the case of Schneiderian membrane perforation during maxillary sinus floor elevation, grafting material displacement into the sinus cavity may accidentally occur. If the surgeon notices any biomaterial dislodgement, the grafting procedure should be immediately interrupted and disseminated particles should be carefully removed from the sinus cavity, by using sinus curettes and Volkmann spoons, irrigation with saline solution and surgical suction. Prompt individuation of graft displacement and effective cleaning procedures are relatively easy during sinus floor elevation with lateral technique: if the same adverse event occurs during the transcrestal approach, it is necessary to extend the mucoperiosteal flap in order to clean properly the sinus cavity through a lateral antrostomy. Moreover, due to the limited intraoperative visibility during transcrestal sinus augmentation, both membrane perforation and graft dispersion can often remain undetected during surgery, being diagnosed only with postoperative radiographs.

Most of the grafting materials commonly used in maxillary sinus floor elevation are in granular form, with diameters varying from $0.25-1 \mathrm{~mm}$ ("small" particles) to 1-2 mm ("large" particles). Additionally, these bone substitutes generally present a very slow resorption rate. Allografts, xenografts and synthetic hydroxyapatite have been histologically detected in the regenerated tissue up to 20 years after the grafting procedure $[13,14,26,27]$.

Pang and coworkers [28] demonstrated that mucociliary system action can effectively transport towards maxillary ostium even relatively heavy foreign bodies, against gravitational force. Therefore, dislodged graft granules may be conveyed and packed through maxillary ostium and ethmoidal infundibulum, compromising OMC patency [16]. OMC patency is an important factor to preserve maxillary sinus homeostasis: ostium occlusion was regarded as one of the key elements in the development of recurrent sinus disease $[29,30]$. The importance of OMC dysfunction in sinus disease pathogenesis is indirectly confirmed by the widespread diffusion of FESS as the surgical treatment of choice to restore normal sinus function in case of chronic sinusitis [31].

In the two cases of graft displacement described in the present report, a xenogeneic bone substitute in gel form was used to elevate the Schneiderian membrane and fill the subantral space after performing an access to the sinus through the bony floor of the cavity. In both cases, the clinician did not identify membrane perforation and graft displacement during surgery, but the diagnosis was made by postoperative radiographs, confirming the low surgeon sensitivity in detecting perforation during the transcrestal approach $[32,33]$. CBCT taken 2 months after surgery showed significant sinus membrane hypertrophy: this finding is very common after sinus floor elevation surgery, due to momentary alterations of ciliary beat frequency occurring as a consequence of surgical trauma. It was suggested that more than 3 months may be necessary for full functional recovery of the respiratory epithelium [34]. The presence of graft particles disseminated within the sinus cavity could likely contribute to more intense and prolonged inflammatory phase: furthermore, the persistence of foreign bodies may represent a trigger factor leading to acute or chronic sinusitis [35]. In the present report, no naso-sinusal symptoms were reported by the two patients during the entire healing period and CBCTs taken 6 months after surgery showed completely healed sinus membrane and no evidence of residual graft into the sinus cavity. The pasty consistency of the collagen gel and the stoichiometric dimensions of the micronized graft particles $(\sim 300 \mu \mathrm{m})$ could likely have facilitated muco-ciliary transport and easy clearance of the disseminated material through maxillary ostium with reduced risk of OMC obstruction. Due to sinus membrane postoperative hypertrophy, OMC mean diameter significantly decreases in the first six months after maxillary sinus floor elevation, varying from 1.1 to $1.8 \mathrm{~mm}$ [36]: for this reason, granules with diameter up to $2 \mathrm{~mm}$ ("large size" grafts) should be avoided in sinus lift procedures for the risk of immediate OMC obliteration in case of accidental dissemination.

These preliminary data seem promising in order to prevent severe complications related to compromised OMC patency in case of accidental displacement of grafting material 
into the sinus cavity during sinus floor elevation. Further studies are needed for large-scale evaluation of the clinical behavior of this grafting material in this specific application.

Author Contributions: Conceptualization, C.S. and F.B.; methodology, F.B., T.L. and C.S.; project administration, F.B.; supervision, T.L.; writing—original draft, C.S.; writing-review and editing, F.B. and T.L. All authors have read and agreed to the published version of the manuscript.

Funding: This research received no external funding.

Institutional Review Board Statement: Not applicable.

Informed Consent Statement: Written informed consent has been obtained from the patients to publish this paper.

Data Availability Statement: Data is contained within this article.

Conflicts of Interest: The authors declare no conflict of interest.

\section{References}

1. Stacchi, C.; Andolsek, F.; Berton, F.; Perinetti, G.; Navarra, C.O.; Di Lenarda, R. Intraoperative complications during sinus floor elevation with lateral approach: A systematic review. Int. J. Oral Maxillofac. Implant. 2017, 32, e107-e118. [CrossRef]

2. Tan, W.C.; Lang, N.P.; Zwahlen, M.; Pjetursson, B.E. A systematic review of the success of sinus floor elevation and survival of implants inserted in combination with sinus floor elevation. Part II: Transalveolar technique. J. Clin. Periodontol. 2008, 35, 241-254. [CrossRef]

3. Boyacıgil, D.U.; Er, N.; Karaca, Ç.; Koç, O. The effect of residual bone height and membrane thickness on sinus membrane perforation in crestal sinus grafting: A prospective clinical study. Int. J. Oral Maxillofac. Surg. 2021, 50, 251-257. [CrossRef]

4. Hernández-Alfaro, F.; Torradeflot, M.M.; Marti, C. Prevalence and management of Schneiderian membrane perforations during sinus-lift procedures. Clin. Oral Implant. Res. 2008, 19, 91-98. [CrossRef] [PubMed]

5. Robiony, M.; Tenani, G.; Sbuelz, M.; Casadei, M. A simple method for repairing membrane sinus perforation. Open J. Stomatol. 2012, 2, 348-351. [CrossRef]

6. Falah, M.; Srouji, S. Use of buccal fat pad for closure of perforation and graft material in a maxillary sinus elevation procedure: A preliminary study. Int. J. Oral Maxillofac. Implant. 2016, 31, 842-848. [CrossRef] [PubMed]

7. Aricioglu, C.; Dolanmaz, D.; Esen, A.; Isik, K.; Avunduk, M.C. Histological evaluation of effectiveness of platelet-rich fibrin on healing of sinus membrane perforations: A preclinical animal study. J. Cranio-Maxillofac. Surg. 2017, 45, 1150-1157. [CrossRef]

8. Barbu, H.M.; Iancu, S.A.; Jarjour Mirea, I.; Mignogna, M.D.; Samet, N.; Calvo-Guirado, J.L. Management of Schneiderian membrane perforations during sinus augmentation procedures: A preliminary comparison of two different approaches. J. Clin. Med. 2019, 8, 1491. [CrossRef]

9. Proussaefs, P.; Lozada, J.L.; Kim, J.; Rohrer, M. Repair of the perforated sinus membrane with a resorbable collagen membrane: A human study. Int. J. Oral Maxillofac. Implant. 2004, 19, 413-420.

10. Danesh-Sani, S.A.; Engebretson, S.P.; Janal, M.N. Histomorphometric results of different grafting materials and effect of healing time on bone maturation after sinus floor augmentation: A systematic review and meta-analysis. J. Periodontal Res. 2017, 52, 301-312. [CrossRef]

11. Starch-Jensen, T.; Mordenfeld, A.; Becktor, J.P.; Jensen, S.S. Maxillary sinus floor augmentation with synthetic bone substitutes compared with other grafting materials: A systematic review and meta-analysis. Implant Dent. 2018, 27, 363-374. [CrossRef] [PubMed]

12. Raghoebar, G.M.; Onclin, P.; Boven, G.C.; Vissink, A.; Meijer, H.J.A. Long-term effectiveness of maxillary sinus floor augmentation: A systematic review and meta-analysis. J. Clin. Periodontol. 2019, 46, 307-318. [CrossRef] [PubMed]

13. Traini, T.; Piattelli, A.; Caputi, S.; Degidi, M.; Mangano, C.; Scarano, A.; Perrotti, V.; Iezzi, G. Regeneration of human bone using different bone substitute biomaterials. Clin. Implant Dent. Relat. Res. 2015, 17, 150-162. [CrossRef]

14. Stacchi, C.; Berton, F.; Fiorillo, L.; Nicolin, V.; Lombardi, T.; Cicciù, M.; Di Lenarda, R. Fresh frozen allogeneic bone block in maxillary sinus floor elevation: Histomorphometric analysis of a bone specimen retrieved 15 years after grafting procedure. Appl. Sci. 2019, 9, 1119. [CrossRef]

15. Felisati, G.; Saibene, A.M.; Lenzi, R.; Pipolo, C. Late recovery from foreign body sinusitis after maxillary sinus floor augmentation. BMJ Case Rep. 2012, 2012, bcr2012007434. [CrossRef]

16. Hunter, W.L.; Bradrick, J.P.; Houser, S.M.; Patel, J.B.; Sawady, J. Maxillary sinusitis resulting from ostium plugging by dislodged bone graft: Case report. J. Oral Maxillofac. Surg. 2009, 67, 1495-1498. [CrossRef] [PubMed]

17. Testori, T.; Drago, L.; Wallace, S.S.; Capelli, M.; Galli, F.; Zuffetti, F.; Parenti, A.; Deflorian, M.; Fumagalli, L.; Weinstein, R.L.; et al. Prevention and treatment of postoperative infections after sinus elevation surgery: Clinical consensus and recommendations. Int. J. Dent. 2012, 2012, 365809. [CrossRef] 
18. Chiapasco, M.; Felisati, G.; Maccari, A.; Borloni, R.; Gatti, F.; Di Leo, F. The management of complications following displacement of oral implants in the paranasal sinuses: A multicenter clinical report and proposed treatment protocols. Int. J. Oral Maxillofac. Surg. 2009, 38, 1273-1278. [CrossRef]

19. Santagata, M.; Guariniello, L.; Rauso, R.; Tartaro, G. Immediate loading of dental implant after sinus floor elevation with osteotome technique: A clinical report and preliminary radiographic results. J. Oral Implantol. 2010, 36, 485-489. [CrossRef] [PubMed]

20. Lopez, M.A.; Lico, S.; Casale, M.; Ormanier, Z.; Carinci, F. The use of various biomaterials in computer-guided crestal sinus lift procedures. A report on two case studies with volume comparison. Oral Implantol. 2016, 9, 89-97.

21. Lopez, M.A.; Casale, M.; Candotto, V.; Papalia, R.; Bressi, F.; Carinci, F. The use of hyaluronic acid as a support of two different micronized biomaterials in crestal sinus lift procedures. A report on two case studies with volume comparison. J. Biol. Regul. Homeost. Agents 2017, 31, 129-138.

22. Cossellu, G.; Farronato, G.; Farronato, D.; Ceschel, G.; Angiero, F. Space-maintaining management in maxillary sinus lifting: A novel technique using a resorbable polymeric thermo-reversible gel. Int. J. Oral Maxillofac. Surg. 2017, 46, 648-654. [CrossRef] [PubMed]

23. Stacchi, C.; Lombardi, T.; Ottonelli, R.; Berton, F.; Perinetti, G.; Traini, T. New bone formation after transcrestal sinus floor elevation was influenced by sinus cavity dimensions: A prospective histologic and histomorphometric study. Clin. Oral Implant. Res. 2018, 29, 465-479. [CrossRef] [PubMed]

24. Stacchi, C.; Spinato, S.; Lombardi, T.; Bernardello, F.; Bertoldi, C.; Zaffe, D.; Nevins, M. Minimally invasive management of implant-supported rehabilitation in the posterior maxilla, Part II. Surgical techniques and decision tree. Int. J. Periodontics Restor. Dent. 2020, 40, e95-e102. [CrossRef] [PubMed]

25. Cosci, F.; Luccioli, M. A new sinus lift technique in conjunction with placement of 265 implants: A 6-year retrospective study. Implant Dent. 2000, 9, 363-368. [CrossRef]

26. Mangano, C.; Piattelli, A.; Perrotti, V.; Iezzi, G. Dense hydroxyapatite inserted into postextraction sockets: A histologic and histomorphometric 20-year case report. J. Periodontol. 2008, 79, 929-933. [CrossRef]

27. Valentini, P.; Bosshardt, D.D. 20-year follow-up in maxillary sinus floor elevation using bovine-derived bone mineral: A case report with histologic and histomorphometric evaluation. Int. J. Oral Maxillofac. Implant. 2018, 33, 1345-1350. [CrossRef] [PubMed]

28. Pang, K.P.; Siow, J.K.; Tan, H.M. Migration of a foreign body in the maxillary sinus illustrating natural mucociliary action. Med. J. Malays. 2005, 60, 383-385.

29. Slavin, R.G.; Spector, S.L.; Bernstein, I.L. The diagnosis and management of sinusitis: A practice parameter update. J. Allergy Clin. Immunol. 2005, 116, S13-S47. [CrossRef]

30. Arikan, O.K.; Onaran, Z.; Muluk, N.B.; Yilmazbaş, P.; Yazici, I. Enophthalmos due to atelectasis of the maxillary sinus: Silent sinus syndrome. J. Craniofacial Surg. 2009, 20, 2156-2159. [CrossRef] [PubMed]

31. Khalil, H.S.; Nunez, D.A. Functional endoscopic sinus surgery for chronic rhinosinusitis. Cochrane Database Syst. Rev. 2006, 3, CD004458. [CrossRef]

32. Berengo, M.; Sivolella, S.; Majzoub, Z.; Cordioli, G. Endoscopic evaluation of the bone-added osteotome sinus floor elevation procedure. Int. J. Oral Maxillofac. Surg. 2004, 33, 189-194. [CrossRef]

33. Elian, S.; Barakat, K. Crestal endoscopic approach for evaluating sinus membrane elevation technique. Int. J. Implant. Dent. 2018, 4, 15. [CrossRef] [PubMed]

34. Kim, Y.M.; Lee, C.H.; Won, T.B.; Kim, S.W.; Kim, J.W.; Rhee, C.S.; Min, Y.G. Functional recovery of rabbit maxillary sinus mucosa in two different experimental injury models. Laryngoscope 2008, 118, 541-545. [CrossRef] [PubMed]

35. Vidal, F.; Coutinho, T.M.; Carvalho Ferreira, D.; Souza, R.C.; Gonçalves, L.S. Odontogenic sinusitis: A comprehensive review. Acta Odontol. Scand. 2017, 75, 623-633. [CrossRef] [PubMed]

36. Sakuma, S.; Ferri, M.; Imai, H.; Mesa, N.F.; Victorio, D.J.B.; Alccayhuaman, K.A.A.; Botticelli, D. Involvement of the maxillary sinus ostium (MSO) in the edematous processes after sinus floor augmentation: A cone-beam computed tomographic study. Int. J. Implant Dent. 2020, 6, 35. [CrossRef] 